\title{
POINTWISE REGULARITY ASSOCIATED WITH FUNCTION SPACES AND MULTIFRACTAL ANALYSIS
}

\author{
STÉPHANE JAFFARD \\ Laboratoire d'Analyse et de Mathématiques Appliquées, Université Paris XII \\ 61 Avenue du Général de Gaulle, 94010 Créteil Cedex, France \\ E-mail: jaffard@univ-paris12.fr
}

This paper is dedicated to Professor Zbigniew Ciesielski on the occasion of his seventieth anniversary

\begin{abstract}
The purpose of multifractal analysis of functions is to determine the Hausdorff dimensions of the sets of points where a function (or a distribution) $f$ has a given pointwise regularity exponent $H$. This notion has many variants depending on the global hypotheses made on $f$; if $f$ locally belongs to a Banach space $E$, then a family of pointwise regularity spaces $C_{E}^{\alpha}\left(x_{0}\right)$ are constructed, leading to a notion of pointwise regularity with respect to $E$; the case $E=L^{\infty}$ corresponds to the usual Hölder regularity, and $E=L^{p}$ corresponds to the $T_{\alpha}^{p}\left(x_{0}\right)$ regularity of Calderón and Zygmund. We focus on the study of the spaces $T_{\alpha}^{p}\left(x_{0}\right)$; in particular, we give their characterization in terms of a wavelet basis and show their invariance under standard pseudodifferential operators of order 0 .
\end{abstract}

1. Introduction. Large classes of signals exhibit a very irregular behavior. In the wildest situations, this irregularity may follow different regimes, and can switch from one regime to another almost instantaneously. Precise recordings of turbulence data (which became available at the beginning of the 80s) showed that turbulence falls in this category. The techniques of multifractal signal analysis have been specifically designed to analyze such behaviors. Initially developed in the mid 80s in the context of turbulence analysis, they were applied successfully to a large range of signals, including traffic data (cars and internet), stock market prices, speech signals, texture analysis, DNA sequences, heartbeat intervals,... (see [1] and references therein).

In such applications, one is interested in analyzing signals whose regularity may widely change from point to point. This instability usually makes the task of determining the pointwise regularity very difficult numerically. However, points with a given regularity

2000 Mathematics Subject Classification: 26A15, 26A30.

The paper is in final form and no version of it will be published elsewhere. 
exponent often form fractal sets; therefore, one is not interested in determining the exact value of the regularity at every point but rather in extracting some relevant information concerning the size and geometry of these fractal sets. Mathematically, the purpose of the multifractal analysis of a function is to determine their Hausdorff dimensions. This motivation indicates why the term "multifractal" was introduced: To each regularity exponent corresponds a fractal set. Therefore, if this exponent takes values in a continuum (say, an interval of non-empty interior), then one deals with a noncountable number of fractals, and the term multifractal is then fully justified.

The definition we gave has to be made more precise: What is the notion of regularity which is used? How can one compute such quantities? Let us recall the different possible definitions which have been proposed up to now. Historically, the first definition of pointwise regularity which was used is the Hölder regularity.

Definition 1 . Let $f: \mathbb{R}^{d} \rightarrow \mathbb{R}$, be a locally bounded function, $x_{0} \in \mathbb{R}^{d}$ and $\alpha \geq 0$. Then $f \in C^{\alpha}\left(x_{0}\right)$ if there exist $R>0, C>0$, and a polynomial $P$ of degree less than $\alpha$ such that

$$
\text { if }\left|x-x_{0}\right| \leq R \text {, then }\left|f(x)-P\left(x-x_{0}\right)\right| \leq C\left|x-x_{0}\right|^{\alpha} .
$$

The Hölder exponent of $f$ at $x_{0}$ is $h_{f}\left(x_{0}\right)=\sup \left\{\alpha: f \in C^{\alpha}\left(x_{0}\right)\right\}$.

The Hölder exponent supplies a definition of pointwise regularity which, in many cases, is not pertinent; here are two examples of such situations (among several others):

- Mathematical results concerning multifractal analysis based on the Hölder exponent necessarily make the assumption that $f$ is continuous, see [8]; in many situations, one wishes to analyse discontinuous functions; an important case in applications is natural images which, because of the occlusion phenomenon (one object is partially hidden by another), always present discontinuities. There exists other settings where one has to consider non-locally bouded functions (for instance in the study of fully developed turbulence, see [1]).

- Let $\Omega \subset \mathbb{R}^{d}$ be a domain of $\mathbb{R}^{d}$ with a fractal boundary. A possible way to perform a multifractal analysis of $\Omega$ consists in associating to its characteristic function $1_{\Omega}$ a pointwise regularity exponent. The Hölder exponent is clearly not the right tool since, in this case, it can take only two values: 0 on the boundary $\partial \Omega$ and $+\infty$ elsewhere.

A third motivation, based on "robustness" requirements, will be detailed in the following section; these numerical stability requirements are related to the fact that the condition $f \in C^{\alpha}\left(x_{0}\right)$ is not invariant under simple pseudodifferential operators of order 0 , and equivalently, cannot be characterized by conditions bearing on the moduli of the wavelet coefficients of $f$.

The main motivation of this paper is to discuss which pointwise regularity criteria should be adopted in particular in the setting of multifractal analysis. In Section 2, we describe the criteria which have been used up to now. In Section 3, we discuss which stability properties they should follow and why. In Section 4 , we prove that the $T_{\alpha}^{p}\left(x_{0}\right)$ condition satisfies these stability requirements, and deduce a wavelet characterization of 
these spaces. In Section 5, we give consequences in multifractal analysis. Some of the results of this paper have been announced in [12].

2. Pointwise regularity spaces. Let $B\left(x_{0}, r\right)$ denote the open ball centered at $x_{0}$ and of radius $r$; the condition $f \in C^{\alpha}\left(x_{0}\right)$ can obviously be rewritten as follows: There exists a polynomial $P$ and constants $C, R>0$ such that

$$
\forall r<R \quad\|(f-P)\|_{L^{\infty}\left(B\left(x_{0}, r\right)\right)} \leq C r^{\alpha} .
$$

Therefore, we can reinterpet $C^{\alpha}\left(x_{0}\right)$ as a condition describing how the $L^{\infty}$ norm of $f$ behaves in small neighbourhoods of $x_{0}$. This point of view has two advantages: We will see that it explains why the $C^{\alpha}\left(x_{0}\right)$ does not have the stability properties mentioned above (it is just a consequence of the fact that $L^{\infty}$ does not possess these properties); furthermore, (2) can be immediately generalized; indeed, one can replace the local $L^{\infty}$ norm by another one. For instance, using the $L^{p}$ norm (for $1 \leq p<\infty$ ) one obtains the following definition introduced by Calderón and Zygmund in 1961, see [4].

Definition 2. Let $p \in[1,+\infty)$; a function $f: \mathbb{R}^{d} \rightarrow \mathbb{R}$ in $L_{l o c}^{p}$ belongs to $T_{\alpha}^{p}\left(x_{0}\right)$ if $\exists R, C>0$ and a polynomial $P$ of degree less than $\alpha$ such that

$$
\forall r \leq R, \quad\left(\frac{1}{r^{d}} \int_{B\left(x_{0}, r\right)}\left|f(x)-P\left(x-x_{0}\right)\right|^{p} d x\right)^{1 / p} \leq C r^{\alpha} .
$$

The $p$-exponent of $f$ at $x_{0}$ is $h_{f}^{p}\left(x_{0}\right)=\sup \left\{\alpha: f \in T_{\alpha}^{p}\left(x_{0}\right)\right\}$.

One immediately checks that this notion is weaker than Hölder regularity: If $f \in$ $C^{\alpha}\left(x_{0}\right)$, then, $\forall p, f \in T_{\alpha}^{p}\left(x_{0}\right)$. The drawbacks of the $C^{\alpha}\left(x_{0}\right)$ criterion of smoothness disappear when one considers this notion of regularity; for instance, the Hölder exponent of a characteristic function $1_{\Omega}$ only takes the value 0 along the boundary of $\Omega$, whereas the $p$-exponent may change from point to point and take any nonnegative value, thus opening the way to a multifractal analysis of domains; furthermore, mathematical results concerning a multifractal analysis based on the $p$-exponent do not require any uniform regularity assumption, see [13].

Though Calderón and Zygmund had different motivations (their purpose was to obtain pointwise regularity results for PDEs), they were already aware that the $T_{\alpha}^{p}\left(x_{0}\right)$ condition has a better behavior with respect to simple properties (such as continuity under the action of pseudo-differential operators).

In the following, we will investigate the $T_{\alpha}^{p}\left(x_{0}\right)$ condition in details. But, for now, let us see how the notion of pointwise regularity can be extended further. It is natural to replace in (1) the space $L^{\infty}$ by an arbitrary function space; let $E$ be a Banach space of distributions (perhaps defined modulo $P_{N}$, the vector space of polynomials of degree at most $N)$ and satisfying $\mathcal{S}_{0} \hookrightarrow E \hookrightarrow \mathcal{S}_{0}^{\prime}\left(\mathcal{S}_{0}\right.$ denotes the Schwartz class of $C^{\infty}$ functions $f$ such that $f$ and all its partial derivatives have fast decay, and all the moments of $f$ vanish); we now assume that $E$ is endowed with a semi-norm, which becomes a norm on $E / P_{N}$. If $B$ is a ball of $\mathbb{R}^{d}$, let

$$
\|f\|_{E, B}=\inf _{f=g \text { on } B}\|g\|_{E} .
$$


Definition 3. Let $E$ be a space of distributions satisfying the above conditions. The two-microlocal space of order $\alpha$ associated with $E$ is the space $\mathcal{C}_{E}^{\alpha}\left(x_{0}\right)$ defined by:

$$
\exists P \text { polynomial, } \exists R, C>0, \forall r \leq R \quad\|f-P\|_{E, B\left(x_{0}, r\right)} \leq C r^{\alpha} .
$$

The pointwise regularity exponent of $f$ at $x_{0}$ associated with $E$ (also called the $E$ exponent) is $h_{f}^{E}\left(x_{0}\right)=\sup \left\{\alpha: f \in C_{E}^{\alpha}\left(x_{0}\right)\right\}$.

REMARKS.

- One cannot give an a priori bound on the degree of $P$ that would only depend on $\alpha$ and would insure the existence and uniqueness of $P$; indeed it clearly depends on the space $E$ chosen, as shown by the case $E=L^{p}$, where one easily checks that the condition $\operatorname{deg}(P)<\alpha+d / p$ is necessary and sufficient to insure this existence and uniqueness.

- The two-microlocal space associated with $L^{\infty}$ is precisely $C^{\alpha}\left(x_{0}\right)$; if $E=L^{p}$, we obtain the space $T_{\alpha+d / p}^{p}\left(x_{0}\right)$.

- The way we introduce pointwise regularity differs from Y. Meyer's (Definition 1.1 of [22]). The space $E$ of [22] corresponds to our space $\mathcal{C}_{E}^{\alpha}\left(x_{0}\right)$. Our motivation here is to emphasisize the duality between the "global" space $E$ (which is usually shift invariant, $L^{p}$ for instance), and the corresponding pointwise regularity space $\mathcal{C}_{E}^{\alpha}\left(x_{0}\right)$, in order to show how properties of the second can be derived from properties of the first.

- Pointwise regularity differs from the notion of local regularity at $x_{0}$ which, for Hölder spaces is defined as follows: $f$ belongs to $C_{l o c}^{s}\left(x_{0}\right)$ if there exists $\varphi$ in $\mathcal{D}\left(\mathbb{R}^{d}\right)$ such that $\varphi\left(x_{0}\right)=1$ and $f \phi \in C^{s}\left(\mathbb{R}^{d}\right)$. This notion can be extended to scales of spaces other than $C^{s}$. For instance, the case of the Besov spaces $B_{p}^{s, p}$ is considered in $[24,27]$.

In order to explain where the terminology of two-microlocal space comes from, we first need to recall the definition of the homogeneous Hölder spaces $\dot{C}^{s}\left(\mathbb{R}^{d}\right)$.

If $0<s<1$ then $f \in \dot{C}^{s}\left(\mathbb{R}^{d}\right)$ if

$$
\exists C>0 \text { such that } \forall x, y \in \mathbb{R}^{d}, \quad|f(x)-f(y)| \leq C|x-y|^{s} .
$$

If $s=1, \dot{C}^{s}\left(\mathbb{R}^{d}\right)$ is the Zygmund class of continuous functions satisfying

$$
\exists C>0 \text { such that } \forall x, y \in \mathbb{R}^{d}, \quad|f(x+y)+f(x-y)-2 f(x)| \leq C|y| .
$$

If $s>1$, then $f \in \dot{C}^{s}\left(\mathbb{R}^{d}\right)$ if for any $d$-uple $\beta$ such that $|\beta|=[s], \partial^{\beta} f \in \dot{C}^{s-[s]}\left(\mathbb{R}^{d}\right)$. Finally, if $s<0$ then the spaces $\dot{C}^{s}\left(\mathbb{R}^{d}\right)$ are defined by recursion on $[s]$ by

$$
f \in \dot{C}^{s}\left(\mathbb{R}^{d}\right) \quad \text { if } f=\partial_{1} f_{1}+\cdots+\partial_{d} f_{d} \text { with } f_{j} \in \dot{C}^{s+1}\left(\mathbb{R}^{d}\right) .
$$

The two-microlocal spaces $C_{x_{0}}^{s, s^{\prime}}$ had been introduced by J.-M. Bony in order to study the propagation of singularities of the solutions of nonlinear evolution equations, see [2]. Y. Meyer showed that these spaces are exactly of the form defined above: Let $s^{\prime}>0$; a distribution $f$ belongs to $C_{x_{0}}^{s,-s^{\prime}}$ if and only if

$$
\exists R, C>0, \forall r \leq R \quad\|f\|_{C^{s-s^{\prime}}, B\left(x_{0}, r\right)} \leq C r^{s^{\prime}},
$$

see $[14,21]$. 
Let us now define the corresponding notions which are relevant in multifractal analysis. The notion of dimension mostly used in multifractal analysis is the Hausdorff dimension. Let us recall its definition. Let $A$ be a subset of $\mathbb{R}^{d}$. For each $\varepsilon>0$, let

$$
M_{\varepsilon}^{d}=\inf _{\mathcal{R}} \sum_{i} \varepsilon_{i}^{d}
$$

where $\mathcal{R}$ denotes a generic covering of $A$ by balls $B_{i}$ of diameter $\varepsilon_{i} \leq \varepsilon$; then

$$
\operatorname{dim}(A)=\sup \left\{d: \lim _{\varepsilon \rightarrow 0} M_{\varepsilon}^{d}=+\infty\right\}=\inf \left\{d: \lim _{\varepsilon \rightarrow 0} M_{\varepsilon}^{d}=0\right\} .
$$

Definition 4. Let $f$ be a distribution which belongs locally to $E$. The E-spectrum of singularities of $f$ is

$$
d_{f}^{E}(H)=\operatorname{dim}\left(\left\{x: h_{f}^{E}(x)=H\right\}\right) .
$$

If $E=L^{\infty}$, this function is simply called the spectrum of singularities of $f$ and is denoted by $d_{f}(H)$; if $E=L^{p}$, it is called the $p$-spectrum of singularities of $f$ and is denoted by $d_{f}^{p}(H)$. Properties of the $p$-spectrum are investigated in [13].

3. Stability conditions. Before investigating general pointwise regularity criteria and the corresponding multifractal formalisms, let us come back to the initial problem we mentioned, i.e. the instability of the $C^{\alpha}\left(x_{0}\right)$ condition. Indeed, the initial motivation of Calderón et Zygmund was to understand how pointwise regularity conditions are transformed in the resolution of elliptic PDEs, and they introduced the $T_{u}^{p}(x)$ spaces because the standard pseudodifferential operators of order 0 are not continuous on $C^{\alpha}\left(x_{0}\right)$, whereas it is the case for the $T_{u}^{p}(x)$ spaces. Let us recall how this deficiency of the $C^{\alpha}\left(x_{0}\right)$ condition can be shown. Consider the simplest possible singular integral operator namely, in dimension 1, the Hilbert transform; it is the convolution with the principal value of $1 / x$, i.e. is defined by

$$
\mathcal{H} f(x)=\lim _{\varepsilon \rightarrow 0} \int_{I_{\varepsilon}(x)} \frac{f(y)}{x-y} d y,
$$

where $I_{\varepsilon}(x)=(-\infty, x-\varepsilon] \cup[x+\varepsilon,+\infty)$. An immediate computation shows that

$$
\mathcal{H}\left(1_{[a, b]}\right)(x)=\log \left|\frac{x-b}{x-a}\right| .
$$

Let now $\left(x_{n}\right)_{n \in \mathbb{N}}$ be a strictly decreasing sequence such that $\lim _{n \rightarrow \infty} x_{n}=0$. We can pick a positive, strictly decreasing sequence $a_{n}$ such that $f=\sum_{n=1}^{\infty} a_{n} 1_{\left[x_{n+1}, x_{n}\right]}$ is arbitrarily smooth at $x_{0}$. Nonetheless, (6) implies that

$$
\mathcal{H} f(x)=\sum_{n=1}^{\infty} a_{n} \log \left|\frac{x-x_{n+1}}{x-x_{n}}\right|=-a_{1} \log \left|x-x_{1}\right|+\sum_{n=1}^{\infty}\left(a_{n}-a_{n+1}\right) \log \left|x-x_{n+1}\right|,
$$

which is not locally bounded near the origin, and therefore cannot have any Hölder regularity there. Note that what we really used here is the fact that the Hilbert transform is not continuous on $L^{\infty}$; the "bad behavior" of the pointwise regularity criterion based on $L^{\infty}$ follows from the corresponding "bad behavior" of $L^{\infty}$. On the opposite side, we will see that the continuity of the Hilbert transform on $L^{p}$ implies its continuity on the $T_{u}^{p}(x)$ spaces, as a consequence of Theorem 4 . 
Besides the study of PDEs, another motivation appeared recently in completely different areas and led to the same concerns. Many signals or images are now stored, denoised or transmitted via their wavelet coefficients, see [19]. Therefore, if one wants to obtain information on the local regularity of signals, one needs to be able to characterize in a robust way their pointwise smoothness by conditions bearing on their wavelet coefficients. Recall that, in dimension 1 , a wavelet basis is of the form $2^{j / 2} \psi\left(2^{j} x-k\right), \quad j, k \in \mathbb{Z}$, where $\psi$ and its derivatives up to order $r$ are continuous and have fast decay (one speaks of $r$-smooth wavelets), for an $r$ picked large enough.

The wavelet coefficients are

$$
c_{j, k}=2^{j} \int f(x) \psi\left(2^{j} x-k\right) d x
$$

(we follow here the standard tradition of using an $L^{1}$ norm in the definition of the wavelet coefficients when dealing with Hölder regularity which will simplify formulas in the following by getting rid of an extra factor $2^{d j / 2}$ that would constantly come up otherwise). Instead of indexing the wavelets and wavelet coefficients by couples $(j, k)$, we will rather use the dyadic intervals $\lambda=\left[k 2^{-j},(k+1) 2^{-j}\right]$, thus writting $c_{\lambda}$ instead of $c_{j, k}$. What can be meant by a characterization "in a robust way"? It is natural to suppose that the criterion used is not too much perturbed if the size of each wavelet coefficient is slightly altered. The following definition encapsulates these features.

Definition 5. Let $\Lambda$ be a discrete set. A norm (or a quasi-norm) on sequences $\left(c_{\lambda}\right)$ indexed by $\Lambda$ is robust if it depends only on the moduli $\left|c_{\lambda}\right|$ and if it is an increasing function in each variable $\left|c_{\lambda}\right|$.

Another natural requirement is that the criterion used does not depend on the particular (smooth enough) wavelet basis which is picked. This implies that the infinite matrices which map a wavelet basis on another one should act in a continuous way on the spaces of sequences thus defined. Since these infinite matrices are matrices of operators which are very closely related to the pseudo-differential operators of order 0 considered by Calderón and Zygmund, see Chapter 7 of [20], we are essentially led back to one of our previous requirements.

Let us now be more specific about these different stability requirements. We keep the discussion in dimension 1 for the sake of simplicity. We have met three different continuity requirements:

- under the action of the Hilbert transform,

- under changes of wavelet bases,

- under the action of pseudodifferential operators of order 0 .

How can such conditions be checked, and what is their hierarchy? It is clear that the first criterion is weaker than the third one. It is also weaker that the second one for the following reason: If the $2^{j / 2} \psi\left(2^{j} x-k\right), j, k \in \mathbb{Z}$ form an orthonormal basis of $L^{2}(\mathbb{R})$, and if $\tilde{\psi}$ denotes the Hilbert transform of $\psi$, then the $2^{j / 2} \tilde{\psi}\left(2^{j} x-k\right), \quad j, k \in \mathbb{Z}$ also form an orthonormal basis of $L^{2}(\mathbb{R})$, simply because the Hilbert transform is an isometry on $L^{2}(\mathbb{R})$, and it commutes with translations and dilations (all these properties follow from the fact that, in the Fourier domain, the Hilbert transform is simply a multiplication 
by $\xi /|\xi|$, which is of modulus one and is homogeneous of degree 0 ). The second and the third conditions do not really compare, but are both implied by a fourth requirement (as a consequence of Theorem 1 below), which is simpler to check in practice, and which we now describe.

We come back to the general $d$-dimensional setting. We start by recalling the form of $d$-dimensional wavelet bases.

Definition 6. An $r$-smooth wavelet basis is composed of $2^{d}-1$ wavelets $\psi^{(i)}$ which belong to $C^{r}$ and satisfy the following properties:

- $\forall i, \forall \alpha$ such that $|\alpha| \leq r, \quad \partial^{\alpha} \psi^{(i)}$ has fast decay,

- The set of functions $2^{d j / 2} \psi^{(i)}\left(2^{j} x-k\right), j \in \mathbb{Z}, k \in \mathbb{Z}^{d}, i \in\left\{1, \ldots, 2^{d}-1\right\}$ is an orthonormal basis of $\left.L^{2}(\mathbb{R})^{d}\right)$.

Thus any function $f$ in $L^{2}\left(\mathbb{R}^{d}\right)$ can be written

$$
f=\sum c_{j, k}^{(i)} \psi^{(i)}\left(2^{j} x-k\right)
$$

where

$$
c_{j, k}^{(i)}=2^{d j} \int f(x) \psi^{(i)}\left(2^{j} x-k\right) d x .
$$

(Note that, in (8) wavelets are not normalized for the $L^{2}$ norm but for the $L^{\infty}$ norm, and, accordingly, the normalisation of the wavelet coefficients is in $L^{1}$ for the same reason as in (7).)

Wavelets will be indexed by a subset of the set of all dyadic cubes as follows: We can consider that $i$ takes values among all dyadic subcubes $\lambda_{i}$ of $[0,1)^{d}$ of width $1 / 2$ except for $[0,1 / 2)^{d}$; thus, the set of indices $(i, j, k)$ can be relabelled using dyadic cubes: $\lambda$ denotes the cube $\left\{x: 2^{j} x-k \in \lambda_{i}\right\}$; we note $\psi_{\lambda}(x)=\psi^{(i)}\left(2^{j} x-k\right.$ ) (an $L^{\infty}$ normalization is used), and $c_{\lambda}=2^{d j} \int \psi_{\lambda}(x) f(x) d x$. We will use the notations $c_{j, k}^{(i)}$ or $c_{\lambda}$ indifferently for wavelet coefficients. Note that the index $\lambda$ indicates where the wavelet is localized; for instance, if the wavelets $\psi^{(i)}$ are compactly supported then $\exists C: \operatorname{supp}\left(\psi_{\lambda}\right) \subset C \lambda$ where $C \lambda$ denotes the cube of same center as $\lambda$ and $C$ times larger; thus the indexation by the dyadic cubes is more than a simple notation: The wavelet $\psi_{\lambda}$ is "essentially" localized around the cube $\lambda$.

We denote by $\Lambda$ the set of all dyadic cubes which are used to index wavelets, and by $\Lambda_{j}$ the subset of $\Lambda$ composed of the dyadic cubes of width $2^{-j}$.

The classes of infinite matrices acting on sequences indexed by $\lambda$ are defined as follows.

Definition 7. Let $\gamma>0$. An infinite matrix $A\left(\lambda, \lambda^{\prime}\right)$ indexed by the dyadic cubes belongs to $\mathcal{M}^{\gamma}$ if

$$
\left|A\left(\lambda, \lambda^{\prime}\right)\right| \leq \frac{C 2^{-\left(\frac{d}{2}+\gamma\right)\left|j-j^{\prime}\right|}}{\left(1+\left(j-j^{\prime}\right)^{2}\right)\left(1+2^{\inf \left(j, j^{\prime}\right)} \operatorname{dist}\left(\lambda, \lambda^{\prime}\right)\right)^{d+\gamma}} .
$$

The following result was proved by Y. Meyer, see [20].

THEOREM 1. If $\gamma>0$, then $\mathcal{M}^{\gamma}$ is an algebra. Furthermore, if $\left(\psi_{\lambda}\right)$ and $\left(\tilde{\psi}_{\lambda}\right)$ are two $r$-smooth wavelet bases, then the matrix $A\left(\lambda, \lambda^{\prime}\right)=\left\langle\psi_{\lambda} \mid \tilde{\psi}_{\lambda^{\prime}}\right\rangle$ belongs to $\mathcal{M}^{\gamma}$ as soon as $\gamma<r$. 
We denote by $\mathcal{O} p\left(\mathcal{M}^{\gamma}\right)$ the space of operators whose matrix on a $\gamma^{\prime}$-smooth wavelet basis (for any $\gamma^{\prime}>\gamma$ ) belongs to $\mathcal{M}^{\gamma}$. This definition makes sense precisely because Theorem 1 implies that this notion does not depend on the (smooth enough) wavelet basis which is used. Pseudodifferential operators of order 0 , such as the Hilbert transform in dimension 1, or the Riesz transforms in higher dimensions, belong to the algebras $\mathcal{O} p\left(\mathcal{M}^{\gamma}\right)$ for any $\gamma$, see Chap. 7 of [20]. In practice, in order to check that a criterion based on wavelet coefficients does not depend on the particular wavelet basis which is chosen, one checks the stronger requirement that it is invariant under the action of $\mathcal{M}^{\gamma}$ for a $\gamma$ large enough.

Definition 8. Let $E$ be a Banach space (or, more generally, a complete vector space with a quasi-norm); $E$ is $\gamma$-stable if the operators of $\mathcal{O} p\left(\mathcal{M}^{\gamma}\right)$ are continuous on $E$.

A sequence $\left(e_{n}\right)_{n \in \mathbb{N}}$ is a basis of $E$ if the following condition holds: For any element $f$ in $E$, there exists a unique sequence $c_{n}$ such that the partial sums $\sum_{n=1}^{N} c_{n} e_{n}$ converge to $f$ in $E$. It is an unconditional basis if furthermore $\exists C>0, \forall N \geq 1$,

$$
\forall\left(\varepsilon_{n}\right)_{n=1, \ldots, N} \text { such that }\left|\varepsilon_{n}\right| \leq 1, \forall\left(c_{n}\right)_{n=1, \ldots, N},\left\|\sum_{n=1}^{N} c_{n} \varepsilon_{n} e_{n}\right\|_{E} \leq C\left\|\sum_{n=1}^{N} c_{n} e_{n}\right\|_{E} .
$$

REMARK. The definition of a basis is usually given in the context of Banach spaces, see $[26,28]$. However it extends immediately to the non-locally convex case, which will be useful in the following.

If the space $E$ is not separable then, of course, it cannot have a basis in the previous sense. In this case, the following weaker notion is used.

Definition 9. Assume that $E$ is the dual of a separable space $F$; then a sequence $\left(e_{n}\right)$ is a weak* basis of $E$ if, $\forall f \in E$, there exists a unique sequence $c_{n}$ such that the partial sums $\sum_{n<N} c_{n} e_{n}$ converge to $f$ in the weak* topology. It is unconditional if furthermore (10) holds.

The properties of weak* bases have been extensively studied by I. Singer, see [26] and references therein. In both settings, if $e_{n}$ is unconditional, then the norm (resp. quasi-norm) on $E$ induces a norm (resp. quasi-norm) on the sequences $\left(c_{n}\right)_{n \in \mathbb{N}}$ by the formula

$$
\left\|\left(c_{n}\right)_{n \in \mathbb{N}}\right\|_{E, w}=\sup _{\left|\varepsilon_{n}\right| \leq 1}\left\|\sum_{n=1}^{\infty} \varepsilon_{n} c_{n} e_{n}\right\|_{E},
$$

where the supremum is taken on all sequences $\left|\varepsilon_{n}\right| \leq 1$; if the basis is unconditional, then the sequence norm thus defined clearly is a robust norm. Note that the sequence space norm associated with a Schauder basis $\left(e_{n}\right)$ is usually defined by

$$
\left\|\left(c_{n}\right)_{n \in \mathbb{N}}\right\|=\sup _{N \in \mathbb{N}}\left\|\sum_{n=1}^{N} c_{n} e_{n}\right\|_{E}
$$

in the unconditional case, the norm we defined is clearly equivalent to this one; we prefer it because it satisfies obviously the robustness property stated in Definition 5. 
The use of specific bases is important in order to understand fine properties of functions, or stochastic processes. A remarkable example is supplied by Z. Ciesielski's decomposition of the Brownian motion on the Schauder basis of $[0,1]$, see [5]; this basis is composed of the "hat functions" $\Delta\left(2^{j} x-k\right)(\Lambda$ is supported on $[0,1]$ where $\Delta(t)=\inf (t, 1-t))$; then,

$$
\text { on }[0,1], \quad B(t)=\xi_{0} t+\sum_{j \geq 0} \sum_{k=0}^{2^{j}-1} \xi_{j, k} 2^{-j / 2} \Delta\left(2^{j} x-k\right),
$$

where $\xi_{0}$ and the $\xi_{j, k}$ are I.I.D. normal centered Gaussians. This decomposition has many remarkable features, and, in particular, allows to derive subtle pointwise properties of functions; for instance it was used by J.-P. Kahane to obtain the existence of slow points of the Brownian motion, see [16].

If $x_{0} \in \mathbb{R}^{d}$, then $\lambda_{j}\left(x_{0}\right)$ denotes the unique dyadic cube of width $2^{-j}$ which contains $x_{0}$. Assume that $E$ is a $\gamma$-stable space and that wavelets are either an unconditional or a weak $^{*}$ unconditional basis of $E$. We can easily deduce from (11) a condition satisfied by the wavelet coefficients of the functions of $C_{E}^{\alpha}\left(x_{0}\right)$; let us denote by $A_{j}^{E}\left(x_{0}\right)$ the quantity

$$
A_{j}^{E}\left(x_{0}\right)=\left\|\left\{d_{\lambda}\right\}_{\lambda \in \Lambda}\right\|_{E, w} \quad \text { where } d_{\lambda}= \begin{cases}c_{\lambda} & \text { if } \lambda \subset 3 \lambda_{j}\left(x_{0}\right), \\ 0 & \text { else. }\end{cases}
$$

Proposition 1. Let $E$ be a $\gamma$-stable complete metric stable space endowed with a norm or a quasi-norm. Let $\left(\psi_{\lambda}\right)_{\lambda \in \Lambda}$ be a compactly supported (smooth enough) wavelet basis. If $f \in C_{E}^{\alpha}\left(x_{0}\right)$, then

$$
\forall j \geq 0, \quad A_{j}^{E}\left(x_{0}\right) \leq C 2^{-\alpha j} .
$$

First note that, if $E$ is $\gamma$-stable, any wavelet basis which is $\gamma^{\prime}$-smooth for a $\gamma^{\prime}>\gamma$ can be used to characterize the norm in $E$, and we can use in particular a compactly supported wavelet basis. Let $D$ be a large enough constant and $g$ be a distribution which coincides with $f(x)-P\left(x-x_{0}\right)$ on $B\left(x_{0}, D 2^{-j}\right)$. The conclusion of Proposition 1 follows by applying the wavelet characterization of $E$ and noticing that, if $D$ is large enough and $\lambda \subset 3 \lambda_{j}\left(x_{0}\right)$, then the corresponding wavelet coefficients of $f$ and $g$ coincide.

If $E=\dot{C}^{s}\left(\mathbb{R}^{d}\right)$, then Y. Meyer proved that the condition given by Proposition 1 characterizes $C_{E}^{\alpha}\left(x_{0}\right)$, see $[14,21]$. Our purpose in the following section is to prove that it is also the case if $E=L^{p}\left(\mathbb{R}^{d}\right)$.

4. The $T_{\alpha}^{p}\left(x_{0}\right)$ spaces. First, let us extend the definition of $T_{\alpha}^{p}\left(x_{0}\right)$ regularity and show how it can be defined for exponents $p$ outside of the range $(1,+\infty)$. If $p=+\infty$ or if $p \leq 1$ then the $L^{p}$ spaces do not have the stability properties that we mentioned above (even the least stringent one, namely the continuity of the Hilbert transform, fails). The "natural extension" of the $L^{p}$ spaces outside of the range $p \in(1,+\infty)$ which has these stability properties is supplied by the space BMO for $p=+\infty$ and by the real Hardy spaces $H^{p}$ for $p \leq 1$. These spaces have a robust wavelet characterization (in the sense supplied by Definition 5) and, as regards Hardy spaces, this characterization has the same form as the wavelet characterization of $L^{p}$. Let us recall the corresponding definitions and characterizations. 
Definition 10. Let $p \leq 1$. A $p$-atom is a function $a(x)$ supported in a ball $B$, and such that

$$
\|a\|_{\infty} \leq \operatorname{Vol}(B)^{-1 / p} \text { and } \forall \alpha \quad \text { such that }|\alpha| \leq d\left(\frac{1}{p}-1\right), \quad \int a(x) x^{\alpha} d x=0 .
$$

A distribution $f$ belongs to $H^{p}$ if it can be written

$$
f=\sum_{n=1}^{\infty} c_{n} a_{n} \text { where each } a_{n} \text { is a } p \text {-atom and } \sum_{n=1}^{\infty}\left|c_{n}\right|^{p}<\infty .
$$

The quasi-norm of $f$ in $H^{p}$ is $\inf \left(\sum_{n=1}^{\infty}\left|c_{n}\right|^{p}\right)$ where the infimum is taken on all possible decompositions of $f$ as a linear combination of $p$-atoms.

If $f \in L_{l o c}^{2}$ and $B$ is a ball, then let

$$
\overline{f_{B}}=\frac{1}{\operatorname{Vol}(B)} \int_{B} f(x) d x
$$

a function $f$ belongs to BMO if $f \in L_{l o c}^{2}$ and satisfies

$$
\|f\|_{B M O}=\sup _{B}\left(\frac{1}{\operatorname{Vol}(B)} \int_{B}\left|f(x)-\overline{f_{B}}\right|^{2} d x\right)^{1 / 2}<\infty,
$$

where the supremum is taken over all balls of $\mathbb{R}^{d}$.

Note that $\|f\|_{B M O}$ only is a semi-norm since BMO is defined modulo constant functions. If $0<p<1$ then $H^{p}$ is not a locally convex space; $\|f\|_{H^{p}}$ is only a quasi-norm, i.e. satisfies

$$
\exists C>0: \quad \forall f, g \quad\|f+g\| \leq C(\|f\|+\|g\|) .
$$

However, $\|f-g\|^{p}$ defines a distance on $H^{p}$.

The wavelet characterizations of these spaces are given by the following theorem, see [20].

THEOREM 2. Let $\psi_{\lambda}$ be a r-smooth wavelet basis with $r>\sup \left(0, d\left(\frac{1}{p}-1\right)\right)$. A distribution $f$ belongs to $L^{p}$ (for $1<p<+\infty$ ) or to $H^{p}$ (for $0<p \leq 1$ ) if and only if its wavelet coefficients satisfy

$$
\left(\sum_{\lambda \in \Lambda}\left|c_{\lambda}\right|^{2} 1_{\lambda}(x)\right)^{1 / 2} \in L^{p}
$$

A function $f$ belongs to BMO if and only if its wavelet coefficients satisfy

$$
\exists C, \forall \lambda, \quad \sum_{\lambda^{\prime} \subset \lambda} 2^{-d j^{\prime}}\left|c_{\lambda^{\prime}}\right|^{2} \leq C \operatorname{Vol}(\lambda) .
$$

Wavelets are unconditional bases of the spaces $L^{p}$ and $H^{p}$, see Chapter 6 of [20]. They cannot be unconditional bases of BMO since this space is not separable; one can check this fact as follows: Let $\psi(x)$ be the Haar wavelet $(\psi$ is supported by $(0,1)$ and takes values 1 on $[0,1 / 2)$ and -1 on $[1 / 2,1))$. Then an easy computation shows that the $B M O$ semi-norm of the function $\sum a_{k} \psi(x-k)$ is equivalent to the $l^{\infty}$ norm of the sequence $a_{k}$; therefore a subspace of $B M O$ is isomorphic to $l^{\infty}$. However, wavelets are weak* unconditional bases of BMO (for the $H^{1}$-BMO duality).

The following definition is the natural extension of $T_{u}^{p}\left(x_{0}\right)$ outside the range $p \in$ $(1, \infty)$ : 
- If $p=+\infty$ we denote by $T_{\alpha}^{\infty}\left(x_{0}\right)$ the space $C_{E}^{\alpha}\left(x_{0}\right)$ with $E=B M O$

- If $0<p \leq 1$ we denote by $T_{\alpha}^{p}\left(x_{0}\right)$ the space $C_{E}^{\alpha}\left(x_{0}\right)$ with $E=H^{p}$.

Let $p \in(0,+\infty]$; then the $p$-exponent of $f$ at $x_{0}$ is $h_{f}^{p}\left(x_{0}\right)=\sup \left\{u: f \in T_{u}^{p}\left(x_{0}\right)\right\}$.

The motivations for considering these new types of pointwise conditions are of a different nature for $p=\infty$ and for $p \leq 1$. If $p=+\infty$, then the $T_{\alpha}^{\infty}\left(x_{0}\right)$ condition is the sharpest condition which is implied by $C^{\alpha}\left(x_{0}\right)$ and can be characterized by a condition bearing on the moduli of the wavelet coefficients of $f$; it is therefore stronger than the two-microlocal conditions $f \in C^{\alpha,-\alpha}\left(x_{0}\right)$ of [8]. In particular, while the two-microlocal condition can be satisfied by distributions which do not coincide with a function in a neighbourhood of $x_{0}$, the $T_{\alpha}^{\infty}\left(x_{0}\right)$ wavelet characterization implies that (3) holds for any $p<\infty$. If $p<1$, then the condition $f \in H_{l o c}^{p}$, allows to deal with singularities such as $\left|x-x_{0}\right|^{-a}$ near $x_{0}$ for $a<d / p$; therefore using arbitrarily small values of $p$ allows to deal with singularities of arbitrarily large exponent $a$, which is needed in several applications, see [1].

The local square functions at $x_{0}$ are

$$
S_{f}\left(j, x_{0}\right)(x)=\left(\sum_{\lambda \subset 3 \lambda_{j}\left(x_{0}\right)}\left|c_{\lambda}\right|^{2} 1_{\lambda}(x)\right)^{1 / 2} .
$$

TheOREM 3. Let $p \in(0, \infty), \alpha>-d / p$ and assume that the wavelet basis used is $r$ smooth with $r>\sup \left(2 \alpha, 2 \alpha+2 d\left(\frac{1}{p}-1\right)\right)$; if $f \in T_{\alpha}^{p}\left(x_{0}\right)$, then $\exists C \geq 0$ such that $\forall j \geq 0$,

$$
\left\|S_{f}\left(j, x_{0}\right)\right\|_{p} \leq C 2^{-j(\alpha+d / p)}
$$

(if $p<1$, then $\|\cdot\|_{p}$ denotes the $L^{p}$ quasi-norm). If $p=+\infty$, this condition becomes

$$
\forall \lambda \subset 3 \lambda_{j}\left(x_{0}\right), \quad\left(\sum_{\lambda^{\prime} \subset \lambda} 2^{-d j^{\prime}}\left|c_{\lambda^{\prime}}\right|^{2}\right)^{1 / 2} \leq C 2^{-d l / 2} 2^{-\alpha j},
$$

where $2^{-l}$ is the width of $\lambda$ and $2^{-j^{\prime}}$ is the width of $\lambda^{\prime}$.

Conversely, if (14) holds (or if (15) holds in the case $p=+\infty$ ) and if $\alpha \notin \mathbb{N}$, then $f \in T_{\alpha}^{p}\left(x_{0}\right)$.

This theorem will be proved in two steps. First, we will show that it holds if the wavelets used are compactly supported, and afterwards, we will show that the elements of $\mathcal{M}^{\gamma}$ are continuous on the space defined by (14); using Theorem 1, this will imply that the characterization holds for any (smooth enough) wavelet basis. If the wavelets are compactly supported, then the direct part of the theorem is just a special case of Proposition 1. Let us now prove the converse part, still in the case of compactly supported wavelets. We can forget the "low frequency component" of $f$ corresponding to $j<0$ in its wavelet decomposition, since its contribution belongs locally to $C^{r}\left(\mathbb{R}^{d}\right)$ (for $r$-smooth wavelets). Let $\Lambda_{j}$ denote the set of dyadic cubes of width $2^{-j}, \Delta_{j} f=\sum_{\lambda \in \Lambda_{j}} c_{\lambda} \psi_{\lambda}$, and let $P_{j}\left(x-x_{0}\right)$ denote the Taylor polynomial of $\Delta_{j} f$ of degree $[\alpha]$ at $x_{0} ;(14)$ or (15) imply that,

$$
\text { if } \operatorname{dist}\left(\lambda, x_{0}\right) \leq D 2^{-j} \text {, then }\left|c_{\lambda}\right| \leq C 2^{-\alpha j} .
$$

Let $\rho>0$ be fixed and let $J$ be defined by $2^{-J} \leq \rho<2 \cdot 2^{-J}$ and $L$ be a constant which will be fixed later, but depends only on the size of the support of the wavelets. If 
$j \leq J+L$, then at most $C$ of the $\psi_{\lambda}$ have a support intersecting $B=B\left(x_{0}, \rho\right)$ and each of them satisfies (16). It follows from Taylor's formula that, if $x \in B$ and $j \leq J+L$, then

$$
\left|\Delta_{j} f(x)-P_{j}\left(x-x_{0}\right)\right| \leq C \rho^{[\alpha]+1} 2^{j([\alpha]+1-\alpha)},
$$

and therefore

$$
\sum_{j=0}^{J+L}\left|\Delta_{j} f(x)-P_{j}\left(x-x_{0}\right)\right| \leq C \rho^{\alpha} .
$$

It follows also from (16) that, if $|k| \leq[\alpha]+1$, then, $\forall j \geq 0,\left|\Delta_{j}^{(k)} f\left(x_{0}\right)\right| \leq 2^{(|k|-\alpha) j}$; therefore the series

$$
P\left(x-x_{0}\right)=\sum_{j=0}^{\infty} P_{j}\left(x-x_{0}\right)=\sum_{j=0}^{\infty} \sum_{|k|<\alpha} \frac{\Delta_{j} f^{(k)}\left(x_{0}\right)}{k !}\left(x-x_{0}\right)^{k}
$$

converges and, if $\left|x-x_{0}\right| \leq \rho$, then

$$
\sum_{j=J+L}^{\infty}\left|P_{j}\left(x-x_{0}\right)\right| \leq C \sum_{j=J+L}^{\infty} \sum_{|k|<\alpha} 2^{(|k|-\alpha) j} \rho^{k} \leq C \rho^{\alpha} .
$$

Let now $g_{J}(x)=\sum_{j=J+L}^{\infty} \Delta_{j} f(x)$; then

$$
\left\|g_{J} 1_{B}\right\| \leq\left\|\sum_{j=J+L}^{\infty} \sum_{\lambda \subset B\left(x_{0}, 2 \rho\right)} c_{\lambda} \psi_{\lambda}\right\|_{p}
$$

where $L$ has been picked large enough so that both functions coincide on $B$. Using the wavelet characterization of $L^{p}$, the right hand side is bounded by

$$
C\left\|\left(\sum_{j=J+L}^{\infty} \sum_{\lambda \subset B\left(x_{0}, 2 \rho\right)}\left|c_{\lambda}\right|^{2} 1_{\lambda}\right)^{1 / 2}\right\|_{p} \leq S_{f}\left(J-L, x_{0}\right) \leq C 2^{-J(\alpha+d / p)} .
$$

The required estimate for $\left\|\left(f-P\left(x-x_{0}\right)\right) 1_{B\left(x_{0}, \rho\right)}\right\|_{p}$ follows immediately from (17), (18) and (19).

The case $p=\infty$ is completely similar.

REMARK. Assume now that $\alpha \in \mathbb{N}$. We can return to the previous proof and subtract only Taylor's expansions of degree $\alpha-1$ (i.e. reproduce exactly the same proof with the convention $[\alpha]=\alpha-1)$. Then all points of the proof run the same except for the derivation of (17); indeed, each term of the sum is now bounded by a constant. It follows that the bound obtained there is $C \rho^{\alpha} \log (1 / \rho)$. Thus, if $\alpha \in \mathbb{N}$ and if (14) holds (or if (15) holds in the case $p=+\infty$ ) then $f$ satisfies $\exists R, C>0$ and a polynomial $P$ of degree less than $\alpha$ such that

$$
\forall r \leq R, \quad\left(\frac{1}{r^{d}} \int\left|f(x)-P\left(x-x_{0}\right)\right|^{p} d x\right)^{1 / p} \leq C r^{\alpha} \log (1 / r) .
$$

In order to end the proof of the theorem, we still have to show that the following theorem is true.

THEOREM 4. If $\gamma>\sup \left(2 \alpha, 2 \alpha+2 d\left(\frac{1}{p}-1\right)\right), \alpha>0$ and $0<p \leq+\infty$, then $T_{\alpha}^{p}\left(x_{0}\right)$ is $\gamma$-stable. 
Proof. We have to prove that, if $A$ is a matrix in $\mathcal{M}^{\gamma}$ and if a sequence $C=\left(c_{\lambda}\right)$ satisfies

$$
\forall j \geq 0, \quad\left\|\left(\sum_{\lambda \subset 3 \lambda_{j}\left(x_{0}\right)}\left|c_{\lambda}\right|^{2} 1_{\lambda}(x)\right)^{1 / 2}\right\|_{p} \leq C 2^{-(\alpha+d / p) j},
$$

then $A C$ satisfies the same estimate. Let us start by introducing a fex notations; if $p>0$, then $E^{p}$ will denote the sequence space of $\left(c_{\lambda}\right)$ indexed by the dyadic cubes and endowed with the norm (or quasi-norm)

$$
\left\|\left(c_{\lambda}\right)\right\|_{p}=\left\|\left(\sum_{\lambda \in \Lambda}\left|c_{\lambda}\right|^{2} 1_{\lambda}(x)\right)^{1 / 2}\right\|_{p} .
$$

If $\mu_{j}\left(=\mu_{j}\left(x_{0}\right)\right)$ denotes the collection of those cubes from $\Lambda$ which are included in $3 \lambda_{j}\left(x_{0}\right)$ and are not included in $3 \lambda_{j+1}\left(x_{0}\right)$, then, the $\mu_{j}$ form a partition of $\Lambda$. Let

$$
d_{\lambda}^{j}= \begin{cases}c_{\lambda} & \text { if } \lambda \subset 3 \lambda_{j}\left(x_{0}\right) \\ 0 & \text { else }\end{cases}
$$

and

$$
e_{\lambda}^{j}= \begin{cases}c_{\lambda} & \text { if } \lambda \in \mu_{j} \\ 0 & \text { else }\end{cases}
$$

we denote by capital letters the corresponding vectors

$$
C=\left(c_{\lambda}\right)_{\lambda \in \Lambda}, \quad D_{j}=\left(d_{\lambda}^{j}\right)_{\lambda \in \Lambda}, \quad E_{j}=\left(e_{\lambda}^{j}\right)_{\lambda \in \Lambda} .
$$

Lemma 1. Condition (21) is equivalent to

$$
\forall j \geq 0, \quad\left\|\left(\sum_{\lambda \in \mu_{j}}\left|c_{\lambda}\right|^{2} 1_{\lambda}(x)\right)^{1 / 2}\right\|_{p} \leq C 2^{-(\alpha+d / p) j} .
$$

Indeed, (22) is weaker than (21) since the sum in (22) bears on less terms. Assume now that (22) holds; note that $D_{j}=\sum_{j^{\prime} \geq j} E_{j}$. If the space considered ( $L^{p}$ or $\left.H^{p}\right)$ is a Banach space (i.e. when $p \geq 1$ ), then

$$
\left\|D_{j}\right\|_{p} \leq \sum_{j^{\prime} \geq j}\left\|E_{j^{\prime}}\right\|_{p} \leq C \sum_{j^{\prime} \geq j} 2^{-(\alpha+d / p) j^{\prime}} \leq C 2^{-(\alpha+d / p) j} .
$$

If $p<1$, one applies the same argument to $\left\|D_{j}\right\|_{p}^{p}$, which satisfies the triangular inequality.

Let us return to the proof of Theorem 4 . Let $A_{\lambda, \lambda^{\prime}}$ be an infinite matrix in $\mathcal{M}^{\gamma}$. We define

$$
\tilde{c}_{\lambda}=\sum_{\lambda^{\prime} \in \Lambda} A_{\lambda, \lambda^{\prime}} c_{\lambda^{\prime}}=\sum_{j^{\prime}} \tilde{c}_{\lambda}^{j^{\prime}} \text { where } \tilde{c}_{\lambda}^{j^{\prime}}=\sum_{\lambda^{\prime} \in \mu_{j^{\prime}}} A_{\lambda, \lambda^{\prime}} c_{\lambda^{\prime}} .
$$

Because of Lemma 1, we only have to estimate the norm of $\tilde{C}=\left(\tilde{c}_{\lambda}\right)$ restricted to $\mu_{j}$; for that, we will estimate the norm of each $\tilde{C}^{j^{\prime}}=\left(\tilde{c}_{\lambda}^{j^{\prime}}\right)$. Let $j$ and $j^{\prime}$ be fixed; if $\left|j-j^{\prime}\right| \leq 3$ then, by assumption,

$$
\left\|\left(c_{\lambda}\right)_{\lambda \in \mu_{j^{\prime}}}\right\|_{p} \leq C 2^{-(\alpha+d / p) j^{\prime}} .
$$

By continuity of the matrices of $\mathcal{M}^{\gamma}$ on the space $L^{p}$ or $H^{p}$, it follows that

$$
\left\|\left(\tilde{c}_{\lambda}^{j^{\prime}}\right)\right\|_{p} \leq C 2^{-(\alpha+d / p) j^{\prime}} \leq C 2^{-(\alpha+d / p) j} .
$$

In order to deal with the case $\left|j-j^{\prime}\right|>3$, we first prove the following lemma. 
Lemma 2. Let $A_{\lambda, \lambda^{\prime}}$ be an infinite matrix in $\mathcal{M}^{\gamma}$. If $\left|j-j^{\prime}\right|>3, \lambda \in \mu_{j}$ and $\lambda^{\prime} \in \mu_{j^{\prime}}$ then

$$
A_{\lambda, \lambda^{\prime}}=2^{-\frac{\gamma}{4}\left|j-j^{\prime}\right|} B_{\lambda, \lambda^{\prime}},
$$

where the matrix $B_{\lambda, \lambda^{\prime}}$ (thus defined for $\lambda \in \mu_{j}$ and $\lambda^{\prime} \in \mu_{j^{\prime}}$ and extended by 0 outside this set of indices) belongs to $\mathcal{M}^{\gamma / 2}$, and its norm in $\mathcal{M}^{\gamma / 2}$ is bounded independently of $j, j^{\prime}$ and $x_{0}$.

Proof. Since the estimates required are symmetric, we can assume that $j \leq j^{\prime}$ (and therefore that $\left.j \leq j^{\prime}-4\right)$.

Denote by $2^{-l}$ the width of $\lambda$ and by $2^{-l^{\prime}}$ the width of $\lambda^{\prime}$. Since $\lambda \in \mu_{j}$ it follows that $l \geq j-1$; for the same reason, $l^{\prime} \geq j^{\prime}-1$.

Assume first that $l \leq \frac{j+j^{\prime}}{2}$; then $\left|l-l^{\prime}\right|=l^{\prime}-l \geq \frac{j-j^{\prime}}{2}-1$ so that

$$
2^{-\left(\frac{d}{2}+\gamma\right)\left|l-l^{\prime}\right|} \leq 2^{\gamma} 2^{-\left(\frac{d}{2}+\frac{\gamma}{2}\right)\left|l-l^{\prime}\right|} 2^{-\frac{\gamma}{2}\left|j^{\prime}-j\right|} ;
$$

hence Lemma 2 holds in this case.

If $l>\frac{j+j^{\prime}}{2}$, since $\lambda \notin 3 \lambda_{j+1}\left(x_{0}\right)$, it follows that $\operatorname{dist}\left(\lambda, x_{0}\right) \geq 2^{-j-1}$; since $\lambda^{\prime} \in$ $3 \lambda_{j+3}\left(x_{0}\right)$, it follows that $\operatorname{dist}\left(\lambda, \lambda^{\prime}\right) \geq 2^{-j-2}$; but $\inf \left(l, l^{\prime}\right)-(j+2) \geq \frac{j^{\prime}+j}{2}-(j+2)=$ $\frac{j^{\prime}-j}{2}-2$. Therefore $2^{\inf \left(l, l^{\prime}\right)} \operatorname{dist}\left(\lambda, \lambda^{\prime}\right) \geq 2^{-\frac{j^{\prime}-j}{2}-2}$ and Lemma 2 follows in this case.

We can now return to the proof of Theorem 4 . If $F=\left(f_{\lambda}\right)$ is a vector indexed by the dyadic cubes, then we denote by $F_{j}$ the vector

$$
\begin{aligned}
\left(F_{j}\right)_{\lambda} & =f_{\lambda} \text { if } \lambda \in \mu_{j} \\
& =0 \text { else. }
\end{aligned}
$$

By hypothesis, $\left\|E_{j}\right\|_{p} \leq C 2^{-(\alpha+d / p) j}$. We want to show that $\left\|(A C)_{j}\right\|_{p} \leq C 2^{-(\alpha+d / p) j}$. For that, we will estimate the size of each $\left(A\left(E_{j^{\prime}}\right)\right)_{j}$.

Since $\left\|E_{j^{\prime}}\right\|_{p} \leq C 2^{-(\alpha+d / p) j^{\prime}}$, by continuity of $B$ on $E^{p}$, it follows that $\left\|B\left(E_{j^{\prime}}\right)\right\|_{p} \leq$ $C 2^{-(\alpha+d / p) j^{\prime}}$. But if $\lambda^{\prime}$ belongs to $\mu_{j^{\prime}}$ and $\lambda$ belongs to $\mu_{j}$, then Lemma 2 implies that $\left\|\left(A\left(E_{j^{\prime}}\right)\right)_{j}\right\|_{p} \leq C 2^{-\frac{\gamma}{4}\left|j-j^{\prime}\right|} 2^{-(\alpha+d / p) j^{\prime}}$, and therefore, if $p \geq 1$, then

$$
\left\|(A C)_{j}\right\| \leq \sum_{j^{\prime}} C 2^{-\frac{\gamma}{4}\left|j-j^{\prime}\right|} 2^{-(\alpha+d / p) j^{\prime}},
$$

which is bounded by $C 2^{-(\alpha+d / p) j}$ as soon as $\gamma / 4>\alpha$. The proof for $p<1$ is similar: One considers \|\|$_{p}^{p}$ to which the triangular inequality applies. The case $p=+\infty$ is similar and left to the reader.

REMARKS. If $p=2$, this characterization boils down to a local $l^{2}$ condition on the wavelet coefficients

$$
\sum_{\lambda^{\prime} \subset 3 \lambda_{j}\left(x_{0}\right)} 2^{-d\left(j^{\prime}-j\right)}\left|c_{\lambda^{\prime}}\right|^{2} \leq C 2^{-(2 \alpha+d) j}
$$

which was previously obtained by Yves Meyer (personal communication) using an alternative proof.

If $p=+\infty$, and if $1 \leq p<+\infty$, then Theorem 3 improves previous results of, respectively, [8] and [13]; up to now, the converse part required a uniform regularity assumption $f \in B_{p}^{\varepsilon, p}$ for an $\varepsilon>0$, which turns out to be unnecessary. Note also that, if $f$ satisfies (15), then $f \in T_{\alpha}^{p}\left(x_{0}\right)$ for any $p<\infty$. This is in sharp contrast with the 
two-microlocal condition obtained in [8] as a consequence of $C^{\alpha}\left(x_{0}\right)$ regularity which does not imply any $T_{u}^{p}\left(x_{0}\right)$ regularity result (or even that $f$ locally coincides with a function).

If $p \neq 2$, then (14) is not a local $l^{p}$ condition on the wavelet coefficients; however, the embeddings between Sobolev and Besov spaces supply the following conditions which are easier to use in practice:

If $p \geq 2$, then $L^{p} \hookrightarrow B_{p}^{0, p}$; thus if $f \in T_{\alpha}^{p}\left(x_{0}\right)$ for $p \geq 2$, then

$$
\sum_{\lambda^{\prime} \subset 3 \lambda_{j}\left(x_{0}\right)} 2^{-d\left(j^{\prime}-j\right)}\left|c_{\lambda^{\prime}}\right|^{p} \leq C 2^{-p \alpha j} .
$$

Similarly, if $p \leq 2$, then $B_{p}^{0, p} \hookrightarrow L^{p}$; thus if

$$
\sum_{\lambda^{\prime} \subset 3 \lambda_{j}\left(x_{0}\right)} 2^{-d\left(j^{\prime}-j\right)}\left|c_{\lambda^{\prime}}\right|^{p} \leq C 2^{-p \alpha j},
$$

then $f \in T_{\alpha}^{p}\left(x_{0}\right)$. Additional results concerning the $T_{\alpha}^{p}$ conditions can be found in $[13,12]$.

5. Implications in multifractal analysis. One cannot expect to compute the spectrum of singularities of an experimental signal by following the algorithm implicit in Definition 4 step by step. Indeed, the computation of a regularity exponent leads to numerical instabilities if it jumps from point to point; the determination of the level sets of a complicated function is also a problem, and finally, computing one Hausdorff dimension involves considering all possible coverings of the corresponding set, which is not numerically feasible... and in the case of a multifractal function, we expect to deal with an infinite number of such sets! The purpose of the multifractal formalism is to derive the spectrum of singularities from quantities effectively computable on experimental signals. Let us consider the setting provided by the $T_{\alpha}^{p}$ regularity, in which case, we wish to obtain the $p$-spectrum. We use the remarkable idea introduced by G. Parisi and U. Frisch in [25] in the setting of Hölder regularity; it was later adapted using wavelets (see $[1,15]$ ), and we present a further extension in the $T_{\alpha}^{p}$ setting: We consider global quantities obtained by averaging the quantity

$$
S_{f}^{\lambda}(p)=\left(\int_{\lambda}\left(\sum_{\lambda^{\prime} \subset \lambda}\left|c_{\lambda^{\prime}}\right|^{2} 1_{\lambda^{\prime}}(x)\right)^{p / 2} d x\right)^{1 / p} .
$$

In order to keep as much information as possible, one actually computes averages of $\left(S_{f}^{\lambda}(p)\right)^{q}$ for all values of the parameter $q$. One obtains thus the $p$-structure function

$$
\Sigma_{j}^{p}(q)=\sum_{\lambda \in \Lambda_{j}}\left(S_{f}^{\lambda}(p)\right)^{q} .
$$

The behavior of these quantities when $j \rightarrow+\infty$ is described by the $p$-scaling function

$$
\eta_{f}^{p}(q)=\liminf _{j \rightarrow+\infty} \frac{\log \left(2^{d\left(\frac{q}{p}-1\right) j} \Sigma_{j}^{p}(q)\right)}{\log \left(2^{-j}\right)} .
$$

Thus $\Sigma_{j}^{p}(q)$ is of the order of magnitude of $2^{-\eta_{f}^{p}(q) j}$ in the limit of small scales. The fundamental idea of the multifractal formalism is to estimate the contribution to $\Sigma_{j}^{p}(q)$ of the points $x_{0}$ where the $p$-exponent takes the value $H$. Indeed, if the cube $\lambda$ contains such a point then, Theorem 3 asserts that $S_{f}^{\lambda}(p) \sim 2^{-H j}$. Coming back to the definition of the 
dimension, we need about $2^{d_{f}^{p}(H) j}$ such cubes to cover the set $\left\{x_{0}: h_{f}^{p}\left(x_{0}\right)=H\right\}$ by cubes of size $2^{-j}$; thus the contribution we look for is, for each value of $j, 2^{-d j} 2^{-d_{f}^{p}(H) j} 2^{-H p j}$ $=2^{-\left(d-d_{f}^{p}(H) j+H p\right) j}$, When $j \rightarrow+\infty$, the contribution given by the smallest possible exponent $d-d_{f}^{p}(H)+H p$ becomes preponderant; thus, we expect that

$$
\eta_{f}^{p}(q)=\inf _{H}\left(d-d_{f}^{p}(H)+H q\right) .
$$

If $d-d_{f}^{p}(H)$ is a convex function, then $-\eta_{f}^{p}(q)$ is the Legendre transform of $d-d_{f}^{p}(H)$ (in the sense of convex functions duality, see Chap. 1.3 of [3]). The inversion formula allows us to recover $d_{f}^{p}(H)$ :

$$
d_{f}^{p}(H)=\inf _{q}\left(H q-\eta_{f}^{p}(q)+d\right) .
$$

This heuristic argument cannot be turned into a mathematical proof, and indeed, there exist counterexamples to (24) even when the spectrum of singularities is concave. When (24) holds, one says that $f$ satisfies the $p$-multifractal formalism; the following result shows that it always yields an upper bound for the spectrum.

THEOREM 5. Let $f \in L_{l o c}^{p}$; then

$$
d_{p}^{f}(H) \leq \inf _{q \neq 0}\left(d-\eta_{f}^{p}(q)+H q\right) .
$$

Sketch of proof. It follows from Theorem 3 that

$$
h_{f}^{p}\left(x_{0}\right)=-\frac{d}{p}+\liminf _{j \rightarrow+\infty}\left(\frac{-1}{j} \log _{2}\left(\sup _{\lambda^{\prime} \in \operatorname{adj}(\lambda)} S_{f}^{\lambda^{\prime}}(p)\right)\right)
$$

where $\operatorname{adj}(\lambda)$ denotes the $3^{d}$ dyadic cubes of same width as $\lambda$ and such that $\bar{\lambda} \cap \bar{\lambda}^{\prime} \neq \emptyset$. The proof of Theorem 5 is exactly the same as the upper bound for the Hölder spectrum given in Section 3.2 of [11], since the only property used in [11] is a result similar to (25) (Theorem 1 of [11]).

Note that, in Theorem 5, no global regularity assumption is needed; this is in contrast with the result of [11], where such an assumption is needed to insure the validity of the formula corresponding to (25) (but not in the proof of the upper bound).

When $p$ is positive, the scaling function can be given a function space interpretation.

Definition 11. Let $s \in \mathbb{R}, p>0$ and $q>0$. The oscillation space $\mathcal{O}_{p}^{s, q}\left(\mathbb{R}^{d}\right)$ is the set of functions $f$ which belong locally to $L^{p}$ and satisfy

$$
\forall j \geq 0, \quad \sum_{\lambda \in \Lambda_{j}}\left(S_{f}^{\lambda}(p)\right)^{q} \leq C 2^{(d-s q) j} .
$$

It follows from this definition that, if $p$ and $q$ are positive, then

$$
\eta_{f}^{p}(q)=\sup \left\{s: f \in \mathcal{O}_{p}^{s / q, q}\right\} .
$$

Spaces of this type have been studied in $[11,13]$. We intend to expose their properties in a forthcoming paper. Note that, initially, oscillation spaces were introduced in a different context: A problem raised by Z. Ciesielski was to determine if the box dimension of the graph of a function can be deduced from the its wavelet coefficients, see [6, 7], and the results of these papers pointed towards a positive answer; A. Deliu and B. Jawerth proposed a formula based on Besov spaces which, unfortunately, turned out to be wrong, 
as shown by A. Kamont and B. Wolnik in [18]. Actually, the answer is given by a particular kind of oscillation spaces defined as follows: let $d_{\lambda}=\sup _{\lambda^{\prime} \subset \lambda}\left|c_{\lambda^{\prime}}\right|$. Then

$$
f \in \mathcal{O}_{\infty}^{s, 1}\left(\mathbb{R}^{d}\right) \quad \text { if } \forall j \geq 0, \quad \sum_{\lambda \in \Lambda_{j}} d_{\lambda} \leq C 2^{(d-s) j} .
$$

Assume that $\exists \varepsilon>0$ such that $f \in C^{\varepsilon}\left(\mathbb{R}^{d}\right)$, and denote by $\delta$ the upper box dimension of the graph of $f$; then

$$
\delta=\sup \left(d, d+1-\sup \left\{s: f \in \mathcal{O}_{\infty}^{s, 1}\right\}\right),
$$

see $[9,10]$. A. Kamont solved the problem raised by Z. Ciesielski completely by showing how to derive the lower box dimension of the graph of a function from its wavelet coefficients, see [17].

Acknowledgements. I am extremely grateful to the anonymous referee for many useful remarks and corrections and for suggesting several improvements in the presentation of this paper.

\section{References}

[1] A. Arneodo, B. Audit, N. Decoster, J.-F. Muzy and C. Vaillant, Wavelet-based multifractal formalism: applications to DNA sequences, satellite images of the cloud structure and stock market data, in: The Science of Disasters, A. Bunde, J. Kropp, H. J. Schellnhuber (eds.), Springer, (2002) 27-102.

[2] J.-M. Bony, Second microlocalization and propagation of singularities for semilinear hyperbolic equations, in: Hyperbolic Equations and Related Topics (Katata/Kyoto, 1984), Academic Press, 1986, 11-49.

[3] H. Brezis, Analyse fonctionnelle, Masson, 1983.

[4] A. P. Caldéron and A. Zygmund, Local properties of solutions of elliptic partial differential equations, Studia Math. 20 (1961), 171-227.

[5] Z. Ciesielski, Hölder conditions for realizations of Gaussian processes, Trans. Amer. Math. Soc. 99 (1961), 403-413.

[6] Z. Ciesielski, Fractal functions and Schauder bases, in: Panoramas of Mathematics, Colloquia 93-94, Banach Center Publ. 34, B. Jakubczyk et al. (eds.), 1995, 47-54.

[7] Z. Ciesielski, Fractal functions and Schauder bases, Comput. Math. Appl. 30 (1995), 283291.

[8] S. Jaffard, Pointwise smoothness, two-microlocalization and wavelet coefficients, Publ. Matem. 35 (1991), 155-168.

[9] S. Jaffard, Sur la dimension de boîte des graphes, C.R.A.S. 326 (1998), 555-560.

[10] S. Jaffard, Oscillation spaces: Properties and applications to fractal and multifractal functions, J. Math. Phys. 39 (1998), 4129-4141.

[11] S. Jaffard, Wavelet techniques in multifractal analysis, in: Fractal Geometry and Applications: A Jubilee of Benoît Mandelbrot; M. Lapidus and M. van Frankenhuijsen (eds.), Proc. Symposia in Pure Mathematics 72, Part 2, AMS, 2004, 91-152.

[12] S. Jaffard, Pointwise regularity criteria, C.R.A.S. 339 (2004), 757-762.

[13] S. Jaffard and C. Mélot, Wavelet analysis of fractal Boundaries, Part 1: Local regularity and Part 2: Multifractal analysis, Comm. Math. Phys. 258 (2005), 513-565. 
[14] S. Jaffard and Y. Meyer, Wavelet methods for pointwise regularity and local oscillations of functions, Mem. Amer. Math. Soc. 123 (1996), 587.

[15] S. Jaffard, Y. Meyer and R. Ryan, Wavelets: Tools for Science and Technology, S.I.A.M., 2001.

[16] J.-P. Kahane, Some Random Series of Functions, Cambridge Univ. Press, 1985.

[17] A. Kamont, On formulae for box dimension of graphs of functions, East J. Approx. 4 (1998), 541-564.

[18] A. Kamont and B. Wolnik, Wavelet expansions and fractal dimension, Constr. Approx. 15 (1998), 97-108.

[19] S. Mallat, A Wavelet Tour of Signal Processing, Academic Press, 1998.

[20] Y. Meyer, Ondelettes et opérateurs, Hermann, 1990.

[21] Y. Meyer, Wavelet analysis, local Fourier analysis and 2-microlocalization, in: Contemporary Mathematics 189 (1995), 393-401.

[22] Y. Meyer, Wavelets, Vibrations and Scalings, Presses de l'Université de Montréal, 1998.

[23] Y. Meyer and H. Xu, Wavelet analysis and chirps, Appl. Comput. Harmon. Anal. 4 (1997), 366-379.

[24] S. Moritoh and T. Yamada, Two-microlocal Besov spaces and wavelets, Rev. Mat. Iberoamericana 20 (2004), 277-283.

[25] G. Parisi and U. Frisch, On the singularity spectrum of fully developped turbulence, in: Turbulence and Predictability in Geophysical Fluid Dynamics, Proceedings of the International Summer School in Physics Enrico Fermi, North-Holland, 1985, 84-87.

[26] I. Singer, Bases in Banach Spaces 1, Springer-Verlag, 1970.

[27] H. Triebel, Wavelet frames for distributions; local and pointwise regularity, Studia Math. 154 (2003), 59-88.

[28] P. Wojtaszczyk, Banach Spaces for Analysts, Cambridge Univ. Press, 1991. 